\title{
SINGULARITIES OF NONLINEAR ELLIPTIC SYSTEMS
}

\author{
DAVID R. ADAMS AND JIE XIAO
}

Dedicated to N. G. Meyers on the occasion of his 80th birthday

\begin{abstract}
Aвstract. Through Morrey's spaces (plus Zorko's spaces) and their potentials/capacities as well as Hausdorff contents/dimensions, this paper estimates the singular sets of nonlinear elliptic systems of the evenordered Meyers-Elcrat type and a class of quadratic functionals inducing harmonic maps.
\end{abstract}

\section{ConTEnTs}

1. Introduction

2. The results

3. The proofs

3.1. Three definitions

3.2. Two lemmas

3.3. One more theorem

3.4. Proof of Theorem 2.1

3.5. Proof of Theorem 2.2

3.6. Proof of Theorem 2.3

References

\section{INTRODUCTION}

In [22], C. B. Morrey discovered a condition satisfied by the first derivatives of weak solutions to certain quasilinear second order systems of elliptic partial differential equations (pde) in domains (connected open sets) $\Omega \subseteq \mathbb{R}^{n}$ that implied everywhere $C^{\alpha}=$ Hölder continuity (of exponent $\alpha$ ) of the solutions throughout $\Omega$, when $n=2$. His condition - now known as the "Morrey condition" - is:

$$
f_{B_{r}\left(x_{0}\right)}\left|\left(\frac{\partial}{\partial x}\right)^{m} u\right|^{p} \leq C r^{-\lambda}
$$

2010 Mathematics Subject Classification. 35J48, 42B37.

JX was supported in part by NSERC of Canada. 
for all open balls $B_{r}\left(x_{0}\right)=\left\{x \in \mathbb{R}^{n}:\left|x-x_{0}\right|<r\right\} \subseteq \Omega ; 0<\lambda \leq n, 1 \leq p<$ $\infty, m \in \mathbb{N}$ (for derivatives of order $m$ ), and $C$ is a positive constant and $f_{E}$ stands for the integral average over $E$ with respect to the Lebesgue measure. Thus was born Morrey's Lemma:

$$
\text { (1.1) } \Longrightarrow u \in C^{\alpha} \quad \text { for } \quad \alpha=m-\frac{\lambda}{p}>0 \text {. }
$$

Notice that in the Sobolev theory with merely $(\partial / \partial x)^{m} u \in L^{p}(\Omega)$, i.e., $\lambda=n$, one generally needs $m-n / p>0$ to achieve Hölder continuity. Thus a significant gain is achieved from the Morrey condition. And we will henceforth say that a function $f$ is a Morrey class function on a domain $\Omega$ if it satisfies (1.1) with $f$ replacing $(\partial / \partial x)^{m} u$. Furthermore, we will say that these functions belong to the Morrey space $L^{p, \lambda}(\Omega)$.

Some what later, De Giorgi in [10], gave an explicit example of a system of elliptic pde that could develop internal singularities provided the dimension of the underlying space exceeds two. This example, often quoted, shows that

$$
u(x)=\left(u^{1}(x), \ldots, u^{n}(x)\right)=\frac{x}{|x|^{\gamma}}=\frac{\left(x_{1}, \ldots, x_{n}\right)}{\left(\sum_{k=1}^{n} x_{k}^{2}\right)^{\gamma / 2}}
$$

is a $W^{1,2}$ (Sobolev space)-solution of

$$
-\left(\left(a_{i j}^{k l}(x, u) u_{x_{i}}^{k}\right)_{x_{j}}=0 \quad \forall l=1, \ldots, n\right.
$$

(summation convention) with

$$
\gamma=\frac{n}{2}\left(1-1 / \sqrt{4(n-1)^{2}+1}\right)
$$

and

$$
a_{i j}^{k l}(x, u)=\delta_{i j} \delta_{k l}+\left(c \delta_{i k}+d b_{i k}(x, u)\right)\left(c \delta_{j l}+d b_{j l}(x, u)\right)
$$

where $c, d$ are two positive constants; $c=n-2, d=n$ and

$$
b_{i k}(x, u)=\frac{x_{i} x_{k}}{|x|^{2}}
$$

in the De Giorgi case. Then, soon after, Giusti-Miranda [16] followed with the $u$ of (1.2) a solution of (1.3) with $\gamma=1, c=1, d=4 /(n-2)$, and

$$
b_{i k}(x, u)=\frac{u^{i} u^{k}}{1+|u|^{2}} \text {. }
$$

And more recently, Koshelev [18] has refined the De Giorgi example by showing that again (1.2) solves (1.3) with

$$
\gamma=1, \quad c=(n-1)^{-1 / 2}\left(1+\frac{(n-2)^{2}}{n-1}\right)^{-1 / 4}, \quad d=\frac{c+c^{-1}}{n-2}
$$


Furthermore, Koshelev's example is extremal in a certain sense; see [8, Chapter 8].

In this paper, we wish to revisit this question of the size of the singular set for such (higher order) systems, specifically the Meyers-Elcrat system [21] and then make some observations concerning some other nonlinear systems, e.g. the harmonic map system [8, 19]. But, the main point we wish to make here, is that a fundamental principal regarding the Morrey theory has gone unnoticed up to now: the Morrey condition can also be used to say something about weak solutions when one is operating below the continuity threshold, i.e., $m-\lambda / p \leq 0,0<\lambda \leq n$. Our results show that one can gain as much as $n-\lambda$ off the apriori dimension estimates of the singular sets in the Morrey case vs. the Sobolev case. And thus with coefficients of the pde satisfying additional regularity - e.g. uniform continuity away from the singular set - then one achieves the so-called partial regularity: the singular set is relatively closed in $\Omega$ and the solution is regular in the compliment (say $C^{\alpha}$ or even $C^{\infty}$, as in the harmonic map system case).

As mentioned, our main study will be the Meyers-Elcrat system of $2 m$ th order quasilinear elliptic equations - given below. However, a comment about our methods should be given here. The underlying Morrey theory need comes from a series of papers by the authors [4, 6, 5, 7], and in particular, from the estimates on the capacities associated with potentials of functions in the Morrey space $L^{p, \lambda}$, i.e., Riesz potentials $I_{\alpha} f(x)=\int|x-y|^{\alpha-n} f(y)$, where generally $f$ has compact support, $0<\alpha<n, n \geq 3$, and the integral is taken with respect to the $n$-dimensional Lebesgue measure. This is a natural extension of the nonlinear potential theory of [3] where $I_{\alpha} f$ plays a central role but for $f \in L^{p}=L^{p, n}$.

\section{THE RESULtS}

One of the main reasons that makes the Meyers-Elcrat system distinctive is that every $W^{m, p}$-solution $u$ has a "reverse Hölder" exponent $q>p$, i.e., $(\partial / \partial x)^{m} u \in L^{q}$ on $\Omega$. This idea originated from the $2^{\text {nd }}$ order case treated earlier by Meyers [20], but in this 1975 paper of Meyers-Elcrat, they rely on a device discovered by Ghering [12] for derivatives of quasi-conformal maps, a device that has since been made into a force in regularity theory for nonlinear elliptic equations by Giaquinta-Giusti [14]. Normally, however, one can not expect to get Hölder continuity from reverse Hölder, though an increase in integrability exponent of solutions often helps, i.e., $u \in L^{r}$ on $\Omega$, for some $r>n p /(n-m p)=$ Sobolev exponent, $m p<n$. Thus, one generally gets Hölder continuity of solutions only when the reverse Hölder exponent $q$ is sufficiently large and/or the Morrey exponent $\lambda$ is sufficiently small. 
The Meyers-Elcrat system is:

$$
\sum_{|\gamma| \leq m}(-1)^{|\gamma|}\left(\frac{\partial}{\partial x}\right)^{\gamma} A_{\gamma}\left(x, D^{m} u\right)=0 \quad \text { on } \quad \Omega
$$

where

$$
A_{\gamma}: \Omega \times \mathbb{R}^{N} \rightarrow \mathbb{R}^{N}, \quad N=\sum_{k=1}^{m} n^{k}
$$

is a Caratheodory function and

$$
\left\{\begin{array}{l}
\sum_{|\gamma| \leq m} A_{\gamma}\left(x, D^{m} u\right)\left(\frac{\partial}{\partial x}\right)^{\gamma} u \geq a_{0}\left|\left(\frac{\partial}{\partial x}\right)^{m} u\right|^{p}, \quad \text { a.e. on } \Omega \\
\left|A_{\gamma}\left(x, D^{m} u\right)\right| \leq M\left|\left(\frac{\partial}{\partial x}\right)^{m} u\right|^{p-1},|\gamma| \leq m, \quad \text { a.e. on } \Omega .
\end{array}\right.
$$

Here, $p \in(1, \infty), a_{0}$ and $M$ are positive constants, and

$$
\left\{\begin{array}{l}
D^{m} u=\left\{\left(\frac{\partial}{\partial x}\right)^{\gamma} u:|\gamma| \leq m\right\} \\
\left(\frac{\partial}{\partial x}\right)^{m} u=\left\{\left(\frac{\partial}{\partial x}\right)^{\gamma} u:|\gamma|=m\right\} \\
\left(\frac{\partial}{\partial x}\right)^{\gamma}=\left(\frac{\partial}{\partial x_{1}}\right)^{\gamma_{1}} \ldots\left(\frac{\partial}{\partial x_{n}}\right)^{\gamma_{n}} \\
\gamma=\left(\gamma_{1}, \ldots, \gamma_{n}\right) \in \mathbb{N}^{n} \\
|\gamma|=\gamma_{1}+\cdots+\gamma_{n}
\end{array}\right.
$$

Our main result is:

Theorem 2.1. Let $\Omega \subset \mathbb{R}^{n}$ be a bounded domain. If $u$ is a $W^{m, p} \cap L^{q}$ solution of (2.1)-(2.2) with $q>n p /(n-m p)$, then $\left|D^{m} u\right|$ belongs to $L^{p, \lambda}(\Omega)$ with $\lambda=(m+n / q) p<n$ and consequently the singular set

$$
\Sigma_{\hat{p}}(u, \Omega)=\mathrm{S}_{\hat{p}}(u, \Omega) \cup \mathrm{T}(u, \Omega)
$$

has Hausdorff dimension $\leq n p / q$. Here $\hat{p}$ equals 1 or $p$, and

$$
\left\{\begin{array}{l}
\mathrm{S}_{\hat{p}}(u, \Omega)=\left\{x_{0} \in \Omega: \lim \sup _{r \rightarrow 0} f_{B_{r}\left(x_{0}\right)}\left|u-f_{B_{r}\left(x_{0}\right)} u\right|^{\hat{p}}>0\right\} \\
\mathrm{T}(u, \Omega)=\left\{x_{0} \in \Omega: \sup _{r>0}\left|f_{B_{r}\left(x_{0}\right) \subseteq \Omega} u\right|=\infty\right\} .
\end{array}\right.
$$

Furthermore, when $q=\infty$, i.e., bounded solutions, then the singular sets have Hausdorff dimension zero, matching the examples of Giusti-Miranda and Koshelev where bounded isolated point singularities can occur. Further, if the coefficients are regular away from the singular set, then they are isolated points; see [13, Chapter IX].

Next, we notice that our methods can be applied to getting estimates of the singular sets for a class of minima for certain quadratic functionals. These functionals with summation convention take the form

$$
\mathcal{J}(u, \Omega)=\int_{\Omega} A_{i j}^{k l}(x, u) u_{x_{k}}^{i} u_{x_{l}}^{j}
$$


with

$$
\left\{\begin{array}{l}
\text { symmetry : } A_{i j}^{k l}(x, u)=A_{j i}^{l k}(x, u) \\
\text { boundedness : }\left|A_{i j}^{k l}(x, u)\right| \leq M \text { for some constant } M>0 \\
\text { ellipticity : } A_{i j}^{k l}(x, u) \xi_{k}^{i} \xi_{l}^{j} \geq a_{0}|\xi|^{2} \text { for some constant } a_{0}>0 \\
\text { Hölder coefficients : } \frac{\left|A_{i j}^{k l}(x, z)-A_{i j}^{k l}\left(x^{\prime}, z^{\prime}\right)\right|}{\left|\left(x-x^{\prime}, z-z^{\prime}\right)\right|^{\beta}} \lesssim 1 \text { for some constant } \beta \in(0,1) \\
\text { splitting coefficients : } A_{i j}^{k l}(x, u)=g_{i j}(x, u) G^{k l}(x)
\end{array}\right.
$$

where $\mathrm{X} \lesssim \mathrm{Y}$ stands for $\mathrm{X} \leq c \mathrm{Y}$ for a constant $c>0$. Our result is:

Theorem 2.2. Let $\Omega \subset \mathbb{R}^{n}$ be a bounded domain. If $u$ is a bounded $W^{1,2}$ minimizer for the functional $\mathcal{J}(u, \Omega)$ with $A_{i j}^{k l}$ satisfying the above, then $|\nabla u| \in L^{2,2}(B)$ for any $B \Subset \Omega$, and consequently all local singular sets (in the sense of [17], say) of such minimizers have Hausdorff dimension zero. Thus if $\mathcal{J}(\cdot, \cdot)$ is the energy functional for harmonic maps into a compact Riemannian manifold with smooth coefficients, then the solutions (the minimizing harmonic maps) have only sets of isolated points as local singular sets.

Nevertheless, the result of Theorem 2.2 applies only to the so-called local or isolated singular sets because one cannot "localize" a singular set of a minimizing harmonic map that extend to the boundary of $\Omega$ (as in [17] - more about this below). This applies to the singularities that arise, for example, with energy minimizing maps that are independent of a variable: $u(x, y)=x /|x|$ with $(x, y) \in \mathbb{R}^{3} \times \mathbb{R}^{n-3}$. Such a $u$ is a minimizer for the harmonic map system with pde

$$
-\Delta_{g} u=A(u)\langle\nabla u, \nabla u\rangle
$$

with quadratic growth on the right side; see [19]. Now the fact that such solutions satisfy

$$
\sup _{B_{r}\left(x_{0}\right) \Subset \Omega} r^{2} f_{B_{r}\left(x_{0}\right)}\left|\left(\frac{\partial}{\partial x} u\right)\right|^{2}<\infty
$$

is well known - it just follows from the "monotone inequality" for minimizing harmonic maps - see [13, Chapter IX] or [19] - because $u$ is in fact a $W^{1,2}$-solution. Thus one can achieve a singular set as large as dimension $n-3$, the maximum allowable for minimizing harmonic maps. Hence for minimizing harmonic maps, all local or isolated singular sets consist of just isolated points, as in [17].

Our final result again relates to minimizing harmonic maps and their singular sets.

Theorem 2.3. Let $\mathbb{B}^{n}$ and $\mathbb{S}^{m-1}$ are the unit ball of $\mathbb{R}^{n}$ and the unit sphere of $\mathbb{R}^{m}$. If $u=\left(u^{1}, \ldots, u^{m}\right)$ is a minimizing harmonic map from $\mathbb{B}^{n}$ to $\mathbb{S}^{m-1}$ and 
$\operatorname{sing}\left(u, \mathbb{B}^{n}\right)$ is the set of all discontinuous points of $u$, then

$$
\sin g\left(u, \mathbb{B}^{n}\right)=\left\{x \in \mathbb{B}^{n}: I_{1}\left(\left|\left(\frac{\partial}{\partial x}\right) u\right|\right)(x)=\infty\right\},
$$

namely, we are saying that bounded singularities of $u$ correspond to unbounded discontinuities of the 1-Riesz potential of the Hilbert-Schmidt norm $|(\partial / \partial x) u|$ of $(\partial / \partial x) u$ determined by:

$$
\left|\left(\frac{\partial}{\partial x}\right) u\right|^{2}=\sum_{i=1}^{m} \sum_{j=1}^{n}\left|\left(\frac{\partial}{\partial x_{j}}\right) u^{i}\right|^{2} .
$$

These last results on minimizing harmonic maps are in sharp contrast to the singular set results that can occur in the Yamabe problem: $-\Delta u=u^{\frac{n+2}{n-2}}$, $u \geq 0$, in $\Omega \subset \mathbb{R}^{n}, n \geq 3$. Here, it has been shown that the largest singular set one can have here, has dimension $(n-2) / 2$ and this can be realized. And on the other hand one can also have local singular sets of the Cantor type along a line in the complement of where the solution is regular; see [23]. And these sets can have dimension positive and arbitrarily small! This can not happen for minimizing harmonic maps.

\section{THE PROOFS}

3.1. Three definitions. We need concepts of the so-called Zorko space, Hausdorff capacity/dimension, and Morrey capacity.

Definition 3.1. Given a domain $\Omega \subseteq \mathbb{R}^{n}$ and $1<p<\infty, 0<\lambda \leq n$, each Morrey space $L^{p, \lambda}$ on $\Omega$ is equipped with the following norm

$$
\|f\|_{L^{p, \lambda}(\Omega)}=\left(\sup _{B_{r}\left(x_{0}\right) \subseteq \Omega} r^{\lambda} f_{B_{r}\left(x_{0}\right)}|f|^{p}\right)^{\frac{1}{p}} .
$$

We say $f \in L_{0}^{p, \lambda}(\Omega)$ (the Zorko space [25]) whenever $f$ can be approximated by $C_{0}^{1}(\Omega)$-functions in the norm $\|\cdot\|_{L^{p, \lambda}(\Omega)}$.

Each Morrey space has its own capacity.

Definition 3.2. For a domain $\Omega \subseteq \mathbb{R}^{n}, 1<p<\infty, 0<\lambda \leq n, 0<\alpha<n$ and $E \subseteq \Omega$, let

$$
C_{\alpha}\left(E ; L^{p, \lambda}(\Omega)\right)=\inf \left\{\|f\|_{L^{p, \lambda}(\Omega)}^{p}: 0 \leq f \in L^{p, \lambda}(\Omega) \& I_{\alpha} f \geq 1_{E}\right\},
$$

where $1_{E}$ stands for the characteristic function of $E$.

According to [4, Theorem 5.3], we know that if $B_{r}\left(x_{0}\right) \subseteq \Omega$ converges to $x_{0}$ then

$$
C_{\alpha}\left(B_{r}\left(x_{0}\right) ; L^{p, \lambda}(\Omega)\right) \approx \begin{cases}r^{\lambda-\alpha p}, & 1<p<\lambda / \alpha \\ (-\ln r)^{-p}, & 1<p=\lambda / \alpha\end{cases}
$$


Here and later on, $\mathrm{X} \approx \mathrm{Y}$ represents that there exists a constant $c>0$ such that $c^{-1} \mathrm{Y} \leq \mathrm{X} \leq c \mathrm{Y}$.

Definition 3.3. The classical $(0, n] \ni d$-dimensional Hausdorff capacity of a set $E \subset \mathbb{R}^{n}$ is defined via:

$$
\Lambda_{d}^{(\infty)}(E)=\inf \sum_{j} r_{j}^{d}
$$

where the infimum is taken over all countable coverings of $E$ by balls $B_{r_{j}}(\cdot)$. Moreover, the Hausdorff dimension of $E$ is decided by

$$
\operatorname{dim}_{H}(E)=\inf \left\{d: \Lambda_{d}^{(\infty)}(E)=0\right\} .
$$

3.2. Two lemmas. Our first lemma indicates that each Morrey space is actually embedded into the intersection of a family of the Zorko spaces:

Lemma 3.4. Let $\Omega \subset \mathbb{R}^{n}$ be a bounded domain. Then

$$
L_{0}^{p, \lambda}(\Omega) \subset L^{p, \lambda}(\Omega) \subset \cap_{\lambda<\mu<n} L_{0}^{p, \mu}(\Omega) .
$$

Proof. The first inclusion of (3.1) follows from Definition 3.1. To validate the second inclusion in (3.1), via setting $f=0$ outside $\Omega$, we may assume that $f$ is in $L^{p, \lambda}\left(\mathbb{R}^{n}\right)=$ the Morrey space $L^{p, \lambda}(\Omega)$ with $\Omega$ replaced by $\mathbb{R}^{n}$, and then $f_{\epsilon}$ is the $\epsilon$-mollifier of $f$, i.e.,

$$
f_{\epsilon}(x)=\phi_{\epsilon} * f(x)=\int_{\mathbb{R}^{n}} \epsilon^{-n} \phi\left(\epsilon^{-1} y\right) f(x-y)
$$

where

$$
\phi \in C_{0}^{\infty}\left(\mathbb{R}^{n}\right) ; 0 \leq \phi \leq 1 ; \int_{\mathbb{R}^{n}} \phi=1 ; \phi_{\epsilon}(x)=\epsilon^{-n} \phi(x / \epsilon) .
$$

For $\lambda<\mu<n$ let $q=(n-\lambda) /(n-\mu)$ and $q^{\prime}=q /(q-1)$. Then

$$
r^{\mu-n} \int_{B_{r}\left(x_{0}\right)}\left|f-f_{\epsilon}\right|^{p}=\left(r^{\lambda-n} \int_{B_{r}\left(x_{0}\right)}\left|f-f_{\epsilon}\right|^{p}\right)^{\frac{1}{q}}\left(\int_{B_{r}\left(x_{0}\right)}\left|f-f_{\epsilon}\right|^{p}\right)^{\frac{1}{q^{\prime}}}
$$

and hence

$$
\left\|f-f_{\epsilon}\right\|_{L^{p, \mu}(\Omega)} \leq\left\|f-f_{\epsilon}\right\|_{L^{p, \lambda}(\Omega)}^{\frac{1}{q}}\left\|f-f_{\epsilon}\right\|_{L^{p}\left(B_{R}(0)\right)}^{\frac{1}{q^{\prime}}}
$$

for some large finite $R>0$. Note that $f_{\epsilon} \rightarrow f$ in $L_{l o c}^{p}$ but at best $f_{\epsilon}$ is bounded in $L^{p, \mu}$; see also Zorko [25]. Therefore, $f \in L_{0}^{p, \mu}(\Omega)$.

The analysis on Page 1649 of [4] gives that if $1<p<\lambda / \alpha$ and $E \subset$ $B_{R}\left(x_{0}\right) \subset \Omega$ then

$$
\Lambda_{n}^{(\infty)}(E)^{\frac{\lambda-\alpha p}{\lambda}} \lesssim \frac{C_{\alpha}\left(E ; L^{p, \lambda}(\Omega)\right)}{R^{\frac{(\lambda-\alpha p)(\lambda-n)}{\lambda}}} \& C_{\alpha}\left(E ; L^{p, \lambda}(\Omega)\right) \lesssim \Lambda_{\lambda-\alpha p}^{(\infty)}(E) .
$$

Geometrically speaking, the last estimates are rough isocapacitary inequalities for the Morrey capacity and the Hausdorff capacity. But, they can be 
improved to the following Morrey-Hausdorff isocapacitary inequalities extending the well-known result for $\lambda=n$; see also [3].

Lemma 3.5. Let $\Omega \subset \mathbb{R}^{n}$ be a bounded domain, $0<\alpha, \lambda<n, 0 \leq \lambda-\alpha p<$ $d \leq n$ and $E \subseteq \Omega$.

(i) If $1<p<\lambda / \alpha$ and $0<q<d p /(\lambda-\alpha p)$, then

$$
\Lambda_{d}^{(\infty)}(E) \lesssim C_{\alpha}\left(E ; L^{p, \lambda}(\Omega)\right)^{\frac{q}{p}} .
$$

(ii) If $1<p=\lambda / \alpha$ and $0<q \leq 1$, then there is a constant $c>0$ such that

$$
\Lambda_{d}^{(\infty)}(E) \lesssim \exp \left(-c C_{\alpha}\left(E ; L^{p, \lambda}(\Omega)\right)^{\frac{q}{p}}\right) \quad \forall d \in(0, n] .
$$

Proof. On the one hand, suppose $v$ is a non-negative Borel measure on $\mathbb{R}^{n}$ obeying

$$
\sup _{\left(r, x_{0}\right) \in(0, \infty) \times \mathbb{R}^{n}} \frac{v\left(B_{r}\left(x_{0}\right)\right)}{r^{d}}<\infty \text { for } n \geq d>\lambda-\alpha p \geq 0 .
$$

According to [5, Theorem 3.1] (cf. [6]), we have:

(i) If $1<p<\frac{\lambda}{\alpha}$ and $0<\lambda<n$, then

$$
\sup _{\|f\|_{L p, \lambda_{(\Omega)}} \leq 1} \int_{\Omega}\left|I_{\alpha} f\right|^{q} d v<\infty \text { for } q<\frac{d p}{\lambda-\alpha p}
$$

and

$$
\sup _{\|f\|_{L p, \lambda_{(\Omega)}} \leq 1} \int_{\Omega} \frac{\left|I_{\alpha} f\right|^{\tilde{p}}}{\left[\ln \left(1+\left|I_{\alpha} f\right|\right)\right]^{\gamma}} d \nu<\infty \quad \text { for } \quad \tilde{p}=\frac{d p}{\lambda-\alpha p} \& \gamma>2 .
$$

(ii) If $1<p=\frac{\lambda}{\alpha}$ and $0<\lambda \leq n$, then there exists a constant $c>0$ such that

$$
\sup _{\|f\|_{L^{p, \lambda}(\Omega)} \leq 1} \int_{\Omega} \exp \left(c\left|I_{\alpha} f\right|^{q}\right) d v<\infty
$$

holds for $(\lambda, q) \in(0, n) \times(0,1]$ or $(\lambda, q)=\left(n, \frac{n}{n-1}\right)$.

On the other hand, [1, Corollary] tells us that under $d \in(0, n]$, one has

$$
\Lambda_{d}^{(\infty)}(E) \approx \sup _{v} v(E)
$$

where the "sup" is taken over all non-negative Borel measures $v$ on $\mathbb{R}^{n}$ with

$$
\sup _{\left(r, x_{0}\right) \in(0, \infty) \times \mathbb{R}^{N}} \frac{v\left(B_{r}\left(x_{0}\right)\right)}{r^{d}}<\infty .
$$

So, the above-recalled facts, plus the definition of $C_{\alpha}\left(E ; L^{p, \lambda}(\Omega)\right)$, derive the iso-capacitary estimates in Lemma 3.5. 
3.3. One more theorem. The following singularity result for the Morrey potentials $I_{\alpha} L^{p, \lambda}(\Omega)$ will be used later on.

Theorem 3.6. Let $\Omega \subset \mathbb{R}^{n}$ be a bounded domain and $f \in L^{p, \lambda}(\Omega)$.

(i) If $1<p<\lambda / \alpha<\mu / \alpha \leq n / \alpha$, then

$$
C_{\alpha}\left(\Sigma_{1}\left(I_{\alpha} f, \Omega\right) ; L^{p, \mu}(\Omega)\right)=0 \quad \& \quad \operatorname{dim}_{H}\left(\Sigma_{1}\left(I_{\alpha} f, \Omega\right)\right) \leq \lambda-\alpha p
$$

(ii) If $1<p=\lambda / \alpha<\mu / \alpha \leq n / \alpha$, then

$$
C_{\alpha}\left(\Sigma_{1}\left(I_{\alpha} f, \Omega\right) ; L^{p, \mu}(\Omega)\right)=0 \quad \& \quad \operatorname{dim}_{H}\left(\Sigma_{1}\left(I_{\alpha} f, \Omega\right)\right)=0 .
$$

Proof. First of all, for $\epsilon>0$ let $f_{\epsilon}=\phi_{\epsilon} * f$ be of the $\epsilon$-mollifier of $f \in L^{p, \lambda}(\Omega)$ and $\mathcal{M}$ denote the Hardy-Littlewood maximal operator.

Next, let us treat $\mathrm{S}_{1}\left(I_{\alpha} f, \Omega\right)$. For $t>0$ set

$$
\mathrm{S}_{1}\left(I_{\alpha} f, \Omega, t\right)=\left\{x_{0} \in \Omega: \limsup _{r \rightarrow 0} f_{B_{r}\left(x_{0}\right)}\left|I_{\alpha} f-f_{B_{r}\left(x_{0}\right)} I_{\alpha} f\right|>t\right\} .
$$

By Lemma 3.4, we see $f \in L_{0}^{p, \mu}(\Omega)$ and then

$$
\begin{aligned}
t \leq & \limsup _{r \rightarrow 0} f_{B_{r}\left(x_{0}\right)}\left|I_{\alpha} f-f_{B_{r}\left(x_{0}\right)} I_{\alpha} f\right| \\
\lesssim & \limsup _{r \rightarrow 0} f_{B_{r}\left(x_{0}\right)}\left|I_{\alpha}\left(f-f_{\epsilon}\right)-f_{B_{r}\left(x_{0}\right)} I_{\alpha}\left(f-f_{\epsilon}\right)\right| \\
& +\limsup _{r \rightarrow 0}\left|I_{B_{r}\left(x_{0}\right)}\left(f_{\epsilon}\right)-f_{B_{r}\left(x_{0}\right)} I_{\alpha}\left(f_{\epsilon}\right)\right| \\
\lesssim & \mathcal{M}\left(I_{\alpha}\left(\left|f-f_{\epsilon}\right|\right)\right)\left(x_{0}\right) \\
\lesssim & I_{\alpha}\left(\mathcal{M}\left(\left|f-f_{\epsilon}\right|\right)\right)\left(x_{0}\right) .
\end{aligned}
$$

By the definition of $C_{\alpha}\left(\cdot ; L^{p, \mu}(\Omega)\right.$ ) and the boundedness of $\mathcal{M}$ on $L^{p, \mu}$ (cf. [9]) with $p>1$ and $\mu>\lambda$, we get

$$
C_{\alpha}\left(\mathrm{S}_{1}\left(I_{\alpha} f, \Omega, t\right) ; L^{p, \mu}(\Omega)\right) \lesssim t^{-p}\left\|f-f_{\epsilon}\right\|_{L^{p, \mu}(\Omega)}^{p} \quad \forall \quad \epsilon>0,
$$

whence finding (via letting $\epsilon \rightarrow 0$ )

$$
C_{\alpha}\left(\mathrm{S}_{1}\left(I_{\alpha} f, \Omega, t\right) ; L^{p, \mu}(\Omega)\right)=0 .
$$

Since $t>0$ is arbitrary, we obtain

$$
C_{\alpha}\left(\mathrm{S}_{1}\left(I_{\alpha} f, \Omega\right) ; L^{p, \mu}(\Omega)\right)=0 .
$$

This, along with Lemma 3.5(i), deduces

$$
\Lambda_{d}^{(\infty)}\left(\mathrm{S}_{1}\left(I_{\alpha} f, \Omega\right)\right)=0 \quad \forall \quad d>\mu-\alpha p \quad \& \quad 0<q<\frac{p(\mu-\alpha p)}{d} .
$$

As a result, letting $\mu \rightarrow \lambda$, we find $\operatorname{dim}_{H}\left(\mathrm{~S}\left(I_{\alpha} f, \Omega\right)\right) \leq \lambda-\alpha p$. In the last estimate, we have used $\lambda>\alpha p$. Nevertheless, when $\lambda=\alpha p$, we still have (3.2) with $\mu>\lambda$, and thereby reaching $\operatorname{dim}_{H}\left(\mathrm{~S}\left(I_{\alpha} f, \Omega\right)\right)=0$. 
Thirdly, we handle the case for T. Set $d\left(x_{0}, \partial \Omega\right)$ be the distance of $x_{0} \in \Omega$ to the boundary $\partial \Omega$ of $\Omega$ and

$$
\mathrm{T}\left(I_{\alpha} f, \Omega, t\right)=\left\{x_{0} \in \Omega: \sup _{0<r<d\left(x_{0}, \partial \Omega\right)}\left|f_{B_{r}\left(x_{0}\right)} I_{\alpha} f\right|>t\right\} .
$$

Then by Lemma 3.4, we get $f \in L_{0}^{p, \mu}(\Omega) \subset L^{p, \mu}(\Omega)$ and

$$
\begin{aligned}
t & <\sup _{0<r<d\left(x_{0}, \partial \Omega\right)}\left|f_{B_{r}\left(x_{0}\right)} I_{\alpha} f\right| \\
& \lesssim \sup _{0<r<d\left(x_{0}, \partial \Omega\right)} f_{B_{r}\left(x_{0}\right)}\left|I_{\alpha}\left(f-f_{\epsilon}\right)\right|+\sup _{0<r<d\left(x_{0}, \partial \Omega\right)} f_{B_{r}\left(x_{0}\right)}\left|I_{\alpha}\left(f_{\epsilon}\right)\right| \\
& \lesssim \mathcal{M}\left(I_{\alpha}\left(\left|f-f_{\epsilon}\right|\right)\right)\left(x_{0}\right)+\mathcal{M}\left(I_{\alpha}\left(f_{\epsilon}\right)\right)\left(x_{0}\right) \\
& \lesssim I_{\alpha}\left(\mathcal{M}\left(\left|f-f_{\epsilon}\right|\right)\right)\left(x_{0}\right)+I_{\alpha}\left(\mathcal{M}\left(f_{\epsilon}\right)\right)\left(x_{0}\right) .
\end{aligned}
$$

Consequently, there is a constant $c>0$ such that

$$
\mathrm{T}\left(I_{\alpha} f, \Omega, t\right) \subseteq \mathrm{T}_{1}\left(I_{\alpha} f, \Omega, t\right) \cup \mathrm{T}_{2}\left(I_{\alpha} f, \Omega, t\right),
$$

where

$$
\left\{\begin{array}{l}
\mathrm{T}_{1}\left(I_{\alpha} f, \Omega, t\right)=\left\{x_{0} \in \Omega: I_{\alpha}\left(\mathcal{M}\left(\left|f-f_{\epsilon}\right|\right)\right)\left(x_{0}\right) \geq \frac{c t}{2}\right\} \\
\mathrm{T}_{2}\left(I_{\alpha} f, \Omega, t\right)=\left\{x_{0} \in \Omega: I_{\alpha}\left(\mathcal{M}\left(f_{\epsilon}\right)\right)\left(x_{0}\right) \geq \frac{c t}{2}\right\} .
\end{array}\right.
$$

A combined use of the definition of $C_{\alpha}\left(\cdot ; L^{p, \mu}(\Omega)\right)$, the boundedness of $\mathcal{M}$ on $L^{p, \mu}$ and $L^{p, \lambda}$ and the easily-verified uniform boundedness of $f \mapsto f_{\epsilon}$ on $L^{p, \mu}(\Omega)$ (cf. [25]) gives

$$
\begin{aligned}
& C_{\alpha}\left(\mathrm{T}\left(I_{\alpha} f, \Omega, t\right) ; L^{p, \mu}(\Omega)\right) \\
& \lesssim C_{\alpha}\left(\mathrm{T}_{1}\left(I_{\alpha} f, \Omega, t\right) ; L^{p, \mu}(\Omega)\right)+C_{\alpha}\left(\mathrm{T}_{2}\left(I_{\alpha} f, \Omega, t\right) ; L^{p, \mu}(\Omega)\right) \\
& \lesssim t^{-p}\left\|f-f_{\epsilon}\right\|_{L^{p, \mu}(\Omega)}^{p}+t^{-p}\left\|f_{\epsilon}\right\|_{L^{p, \mu}(\Omega)}^{p} \\
& \lesssim t^{-p}\left\|f-f_{\epsilon}\right\|_{L^{p, \mu}(\Omega)}^{p}+t^{-p}\|f\|_{L^{p, \mu}(\Omega)}^{p} .
\end{aligned}
$$

Since $\lim _{\epsilon \rightarrow 0}\left\|f-f_{\epsilon}\right\|_{L^{p, \mu}(\Omega)}^{p}=0$, letting $\epsilon \rightarrow 0$ and then $t \rightarrow \infty$, one derives

$$
C_{\alpha}\left(\mathrm{T}\left(I_{\alpha} f, \Omega\right) ; L^{p, \mu}(\Omega)\right)=0 .
$$

This plus Lemma 3.5 yields

$$
\Lambda_{d}^{(\infty)}\left(\mathrm{T}\left(I_{\alpha} f, \Omega\right)\right)=0 \quad \forall \quad d>\mu-\alpha p>\lambda-\alpha p,
$$

whence giving

$$
\operatorname{dim}_{H}\left(\mathrm{~T}\left(I_{\alpha} f, \Omega\right)\right) \leq \lambda-\alpha p .
$$

Now, the above estimates yield the desired results for $\Sigma_{1}=\mathrm{S}_{1} \cup \mathrm{T}$. 
3.4. Proof of Theorem 2.1. The part on $q=\infty$ follows readily from the argument for the case $n p /(n-m p)<q<\infty$. So, it is enough to handle this last case.

The result $\left|D^{m} u\right| \in L^{p, \lambda}(\Omega)$ with $\lambda=(m+n / q) p<n$ follows from the estimate below:

$$
r^{m p} \int_{B_{r / 2}\left(x_{0}\right)}\left|\left(\frac{\partial}{\partial x}\right)^{m} u\right|^{p} \lesssim \int_{B_{r}\left(x_{0}\right)}|u|^{p} \forall x_{0} \in \Omega \& 0<r<d\left(x_{0}, \partial \Omega\right) .
$$

To verify (3.3), we just use the test function $\varphi=\eta^{m p} u$, where $\eta(x)=\psi\left(\frac{x-x_{0}}{r}\right)$ for which

$$
\psi \in C_{0}^{\infty}\left(\mathbb{R}^{n}\right) \quad \& \quad \psi(x)= \begin{cases}1, & x \in B_{r / 2}\left(x_{0}\right) \\ 0, & x \in \mathbb{R}^{n} \backslash B_{r}\left(x_{0}\right) .\end{cases}
$$

This then gives

$$
\begin{aligned}
\int\left|\left(\frac{\partial}{\partial x}\right)^{m} u\right|^{p} \eta^{m p} \lesssim & \int\left|\left(\frac{\partial}{\partial x}\right)^{m} u\right|^{p-1} \eta^{m(p-1)}\left|\left(\frac{\partial}{\partial x}\right)^{m-k} u\right| r^{-(m-k)} \\
& +\int\left|\left(\frac{\partial}{\partial x}\right)^{m} u\right|^{p-1} \eta^{m(p-1)}\left|\left(\frac{\partial}{\partial x}\right)^{j-l} u\right| r^{-(j-l)}
\end{aligned}
$$

for $0<k \leq m$ and $j<m$ with $0 \leq l \leq j$. Then via the Young inequality

$$
a b \leq \frac{\epsilon a^{\theta}}{\theta}+\frac{\epsilon^{\frac{1}{1-\theta}} b^{\theta^{\prime}}}{\theta^{\prime}} \quad \forall \quad a, b, \epsilon>0, \theta>1, \theta^{\prime}=\frac{\theta}{\theta-1},
$$

we get

$$
\begin{aligned}
\int\left|\left(\frac{\partial}{\partial x}\right)^{m} u\right|^{p} \eta^{m p} \leq & \int_{B_{r}\left(x_{0}\right)}\left|\left(\frac{\partial}{\partial x}\right)^{m-k} u\right|^{p} r^{-(m-k) p} \\
& +\int_{B_{r}\left(x_{0}\right)}\left|\left(\frac{\partial}{\partial x}\right)^{j-l} u\right|^{p} r^{-(j-l) p} .
\end{aligned}
$$

Now, applying the Gagliardo-Nirenberg inequality (see e.g. [11]) gives

$$
\int\left|\left(\frac{\partial}{\partial x}\right)^{m} u\right|^{p} \eta^{m p} \lesssim r^{-m p} \int_{B_{r}\left(x_{0}\right)}|u|^{p} .
$$

Next, we prove

$$
\operatorname{dim}_{H}\left(\Sigma_{\hat{p}}(u, \Omega)\right) \leq n p / q .
$$

To reach (3.4), let $f=\left|D^{m} u\right|$ and consider two cases below. 
Case 1: $\hat{p}=p$. Firstly, we establish the following estimate for $\mu \in(\lambda, n]$ and $t>0$ :

$$
C_{m}\left(\left\{x_{0} \in \Omega ;\left(\mathcal{M}\left(I_{m} f\right)^{p}\left(x_{0}\right)\right)^{1 / p}>t\right\} ; L^{p, \mu}(\Omega)\right) \lesssim t^{-p}\|f\|_{L^{p, \mu}(\Omega)}^{p} .
$$

In fact, observe that

$$
\left(\mathcal{M}\left(I_{m} f\right)^{p}\right)^{1 / p} \lesssim I_{m}\left(\mathcal{M} f^{p}\right)^{1 / p}
$$

and so that the left side of (3.5) does not exceed $t^{-p}\left\|\left(\mathcal{M} f^{p}\right)^{1 / p}\right\|_{L^{p, \mu}(\Omega)}^{p}$ by definition of the Morrey capacity. But clearly

$$
\left(r^{\mu} f_{B_{r}\left(x_{0}\right)} \mathcal{M} f^{p}\right) \lesssim\left(r^{\mu(p+\epsilon) / p} f_{B_{r}\left(x_{0}\right)}(\mathcal{M} f)^{\frac{p+\epsilon}{p}}\right)^{\frac{p}{p+\epsilon}}
$$

holds for small number $\epsilon>0$. So

$$
\left\|\mathcal{M} f^{p}\right\|_{L^{p, \mu}(\Omega)} \lesssim\left\|\mathcal{M} f^{p}\right\|_{L^{(p+\epsilon) / p, \mu(p+\epsilon) / p}(\Omega)} \lesssim\|f\|_{L^{p+\epsilon, \mu(p+\epsilon) / p}(\Omega)}
$$

follows from [9]: the maximal function is a bounded operator on the Morrey spaces $L^{(p+\epsilon) / p, \mu(p+\epsilon) / p}$. But applying the reversed Hölder estimates for $f$ from [21], we get

$$
\|f\|_{L^{p+\epsilon, \mu(p+\epsilon) / p(\Omega)}} \lesssim\|f\|_{L^{p, \mu}(\Omega)} .
$$

Thus the desired result (3.5) follows.

Secondly, we need the fact that any $h \in C_{0}^{\infty}\left(\mathbb{R}^{n}\right)$ can be represented as ([2, Lemma 2]):

$$
h(x)= \begin{cases}(-1)^{\frac{k}{2}}\left(\frac{\omega_{n-1} \beta_{0}}{n}\right)^{\frac{2-n}{n}} \int_{\mathbb{R}^{n}} \frac{\nabla^{k} h(y)}{|x-y|^{n-k}}, & k=2,4,6, \ldots \\ (-1)^{\frac{k-1}{2}}\left(\frac{\omega_{n-1} \beta_{0}}{n}\right)^{\frac{2-n}{n}} \int_{\mathbb{R}^{n}} \frac{(x-y) \cdot \nabla^{k} h(y)}{|x-y|^{n-k+1}}, & k=1,3,5, \ldots,\end{cases}
$$

where $\omega_{n-1}=2 \pi^{n / 2} / \Gamma(n / 2)$ is the volume of the boundary $\mathbb{S}^{n-1}$ of the unit ball $\mathbb{B}^{n}$ of $\mathbb{R}^{n}, \Gamma(\cdot)$ is the usual Gamma function,

$$
\nabla^{k} h= \begin{cases}(-\Delta)^{\frac{k}{2}} h, & k=2,4,6, \ldots \\ \nabla(-\Delta)^{\frac{k-1}{2}} h, & k=1,3,5, \ldots\end{cases}
$$

and

$$
\beta_{0}=\beta_{0}(k, n)= \begin{cases}\frac{n}{\omega_{n-1}}\left(\frac{\pi^{\frac{n}{2}} 2^{k} \Gamma\left(\frac{k+1}{2}\right)}{\left.\Gamma \frac{n-k+1}{2}\right)}\right)^{\frac{n}{n-2}}, & k=2,4,6, \ldots \\ \frac{n}{\omega_{n-1}}\left(\frac{\pi^{\frac{n}{2}} 2^{k} \Gamma\left(\frac{k}{2}\right)}{\Gamma\left(\frac{n-k}{2}\right)}\right)^{\frac{n}{n-2}}, & k=1,3,5, \ldots\end{cases}
$$

Now that each component $u^{l}$ of $u$ is in $W^{m, p}(\Omega) \cap L^{q}(\Omega)$. So the representation formula (3.6) for the even orders can extend to $u^{l}$ via the density of $C_{0}^{\infty}(\Omega)$ in $W^{m, p}(\Omega)$ :

$$
u^{l}(x)= \begin{cases}(-1)^{\frac{m}{2}}\left(\frac{\omega_{n-1} \beta_{0}}{n}\right)^{\frac{2-n}{n}} I_{m}\left(f_{m, l}\right), & m=2,4,6, \ldots \\ (-1)^{\frac{m-1}{2}}\left(\frac{\omega_{n-1} \beta_{0}}{n}\right)^{\frac{2-n}{n}} I_{m}\left(f_{m, l}\right), & m=1,3,5, \ldots\end{cases}
$$


where

$$
f_{m, l}(y)= \begin{cases}\nabla^{m} u^{l}(y), & m=2,4, \ldots \\ |x-y|^{-1}(x-y) \cdot \nabla^{m} u^{l}(y), & m=1,3, \ldots .\end{cases}
$$

For simplicity, set $f=f_{m, l}$ and $g=I_{m}\left(f_{m, l}\right)$. An application of $\left|D^{m} u\right| \in$ $L^{p, \lambda}(\Omega)$ implies $f_{m, l} \in L^{p, \lambda}(\Omega)$. Now, we use $0<\epsilon$-mollifier $f_{\epsilon}=\phi_{\epsilon} * f$ of $f$ to obtain that if $t>0$ then

$$
\begin{aligned}
t^{\frac{1}{p}} & <\liminf _{r \rightarrow 0}\left(f_{B_{r}\left(x_{0}\right)}\left|g-f_{B_{r}\left(x_{0}\right)} g\right|^{p}\right)^{\frac{1}{p}} \\
& \lesssim\left(\mathcal{M}\left(I_{m}\left(f-f_{\epsilon}\right)\right)^{p}\left(x_{0}\right)\right)^{\frac{1}{p}} \\
& \lesssim I_{m}\left(\mathcal{M}\left(\left|f-f_{\epsilon}\right|^{p}\right)\right)^{\frac{1}{p}} .
\end{aligned}
$$

According to the definition of $C_{m}\left(\cdot ; L^{p, \mu}(\Omega)\right)$ and (3.5), we have that if

$$
\mathrm{S}_{p}(g, \Omega, t)=\left\{x \in \Omega: \limsup _{r \rightarrow 0} f_{B_{r}\left(x_{0}\right)}\left|g-f_{B_{r}\left(x_{0}\right)} g\right|^{p}>t\right\} .
$$

then

$$
C_{m}\left(\mathrm{~S}_{p}(g, \Omega, t) ; L^{p, \mu}(\Omega)\right) \lesssim t^{-p}\left\|f-f_{\epsilon}\right\|_{L^{p, \mu}(\Omega)}^{p} .
$$

This last estimate, along with (3.1) of Lemma 3.4 ensuring

$$
\lim _{\epsilon \rightarrow 0}\left\|f-f_{\epsilon}\right\|_{L^{p, \mu}(\Omega)}=0 \quad \forall \quad \mu \in(\lambda, n],
$$

yields

$$
C_{m}\left(\mathrm{~S}_{p}(g, \Omega) ; L^{p, \mu}(\Omega)\right)=0 \quad \forall \quad \mu \in(p(m+n / q), n] .
$$

Thirdly, the T-part of Theorem 3.6 is used to give

$$
C_{m}\left(\mathrm{~T}(g, \Omega) ; L^{p, \mu}(\Omega)\right)=0 \quad \forall \quad \mu \in(p(m+n / q), n] .
$$

Now, putting (3.7) and (3.8) together, we find

$$
C_{m}\left(\Sigma_{p}(g, \Omega) ; L^{p, \mu}(\Omega)\right)=0 \quad \forall \quad \mu \in(p(m+n / q), n] .
$$

This plus Lemma 3.5 yields

$$
\Lambda_{d}^{(\infty)}\left(\Sigma_{p}(g, \Omega)\right)=0 \quad \forall \quad d>\epsilon+n p / q \quad \& \quad \epsilon \in(0,1)
$$

thereby deriving $\operatorname{dim}_{H}\left(\Sigma_{p}(g, \Omega)\right) \leq n p / q$, and so (3.4).

Case 2: $\hat{p}=1$. Under this assumption, (3.4) follows from the above argument and Theorem 3.6 with $\lambda=m p+n p / q, \alpha=m$ and $p=p$.

Remark 3.7. The second derivative estimates in the case $m=1$ follow $[8$, Theorem 8.15]. Using the finite difference operator $\Delta_{2} \phi(x)=\phi(x+z)-\phi(x)$, we can prove that the second derivatives of the solution in Theorem 2.1 lie in the Morrey space $L^{p, 2 m+n p / q}$, at least when the coefficients of our pde have derivatives, i.e., $a_{i x_{j}}^{k}=\frac{\partial a_{i}^{k}}{\partial x_{j}} \in L^{2}$ and $a_{i j}^{k l}=\frac{\partial a_{i}^{k}}{\partial p_{j}^{l}}$ satisfies ellipticity 
and are bounded; see [8]. However, this Morrey space estimate does not decrease locally the size of the singular set; it only increases the dimension of the underlying space $\mathbb{R}^{n}$ that would be needed to admit singularities (nonHölder solutions). Now we would need at least $n \geq 5$, to get such a solution.

3.5. Proof of Theorem 2.2. In what follows, suppose $u$ is a $W^{1,2} \cap L^{\infty}$ minimizer of $\mathcal{J}(u, \Omega)$. Clearly, such $u$ is a $W^{1,2} \cap L^{\infty}$ minimizer of $\mathcal{J}(u, B)$ for any ball $B=B_{r}\left(x_{0}\right) \Subset \Omega$. For each $t \in(0,1)$ and $\tau>0$ let

$$
\Phi\left(t, \tau, r, x_{0}\right)=t^{2-n} e^{\tau \tau^{\beta}} \int_{B_{t r}\left(x_{0}\right)} A_{i j}^{k l}(x, u) u_{x_{k}}^{i} u_{x_{l}}^{j} .
$$

According to [15], there is a constant $\tau$ (independent of $x_{0}$ and $t, r$ ) such that $t \mapsto \Phi\left(t, \tau, r, x_{0}\right)$ is an increasing function on the interval $(0,1)$. As a consequence, one has

$$
\Phi\left(t_{1}, \tau, r, x_{0}\right) \leq \Phi\left(t_{2}, \tau, r, x_{0}\right) \quad \text { for } \quad 0<t_{1}<t_{2}<1
$$

This, along with the elliptic condition on $A_{i j}^{k l}$, implies $|\nabla u| \in L^{2,2}(B)$. Of course, the argument for Theorem 2.1 derives $\operatorname{dim}_{H}\left(\Sigma_{2}(u, B)\right)=0$.

Next, suppose $\Sigma_{2}(u, \Omega)$ (which equals $\mathrm{S}_{2}(u, \Omega)$ since $u$ is bounded) is contained properly in a ball $B \subset \Omega$. Then $\operatorname{dim}_{H}\left(\Sigma_{2}(u, \Omega)\right)=0$ follows from the above argument. Without loss of generality we may assume that $B$ is just the unit ball $\mathbb{B}^{n}$. Since $t \mapsto \Phi(t, \tau, 1,0)=: \Psi(t)$ is an increasing function on $(0,1)$, according to [15, (15)] one has

$$
\int_{\partial \mathbb{B}^{n}}|u(r x)-u(s x)|^{2} d H_{n-1}(x) \lesssim \frac{\Psi(r)-\Psi(s)}{\left(\ln \frac{r}{s}\right)^{-1}} \quad \forall 0<s<r<1 .
$$

In the above and below, $d H_{n-1}$ stands for $n-1$ dimensional Hausdorff measure.

If $\left\{x_{\rho}\right\}_{\rho=1}^{\infty}$ is a sequence of points in $\Sigma_{2}(u, \Omega)$, then this sequence has a subsequence, still denoted by $\left\{x_{\rho}\right\}$, that converges to a point $x_{0} \in \mathbb{B}^{n}$ thanks to $\Sigma_{2}(u, \Omega) \Subset \mathbb{B}^{n}$. For simplicity, set $x_{0}$ be just the center of $\mathbb{B}^{n}$, and $r_{\rho}=$ $2\left|x_{\rho}\right|<1$. Then, $\hat{u}(x)=u\left(r_{\rho} x\right)$ is a local minimizer of

$$
\mathcal{J}_{\rho}\left(\hat{u}, \mathbb{B}^{n}\right)=\int_{\mathbb{B}^{n}} \hat{A}_{i j}^{k l}(x, \hat{u}) \hat{u}_{x_{k}}^{i} \hat{u}_{x_{l}}^{j} \quad \text { with } \quad \hat{A}_{i j}^{k l}(x, \hat{u})=A_{i j}^{k l}\left(r_{\rho} x, \hat{u}\right) .
$$

Referring to the argument on [15, Page 52], $\left\{u\left(r_{\rho} x\right)\right\}$ converges weakly in $L^{2}\left(\mathbb{B}^{n}\right)$ to a map $v$ which is a local minimizer of

$$
\mathcal{J}_{0}\left(v, \mathbb{B}^{n}\right)=\int_{\mathbb{B}^{n}} A_{i j}^{k l}(0, v) v_{x_{k}}^{i} v_{x_{l}}^{j}
$$


Moreover, there is a point $y_{0}$ with $\left|y_{0}\right|=1 / 2$ such that $y_{0}$ is a singular point of $v$. Note that (3.9) is satisfied by $\hat{u}$, i.e.,

$$
\int_{\partial \mathbb{B}^{n}}|\hat{u}(r x)-\hat{u}(s x)|^{2} d H_{n-1}(x) \lesssim \frac{\Psi\left(r r_{\rho}\right)-\Psi\left(s r_{\rho}\right)}{\left(\ln \frac{r}{s}\right)^{-1}} \quad \forall 0<s<r<1 .
$$

Letting $\rho \rightarrow \infty$ in (3.10) produces

$$
\int_{\partial \mathbb{B}^{n}}|v(r x)-v(s x)|^{2} d H_{n-1}(x)=0 .
$$

This indicates that $v$ is constant along the segment from the center of $\mathbb{B}^{n}$ to any point in $\partial \mathbb{B}^{n}$. Because $y_{0}$ is a singular point of $v$, one concludes that the segment between the center of $\mathbb{B}^{n}$ and $y_{0}$ is a subset of $\Sigma_{2}\left(v, \mathbb{B}^{n}\right)$, and so that $\operatorname{dim}_{H}\left(\Sigma_{2}\left(v, \mathbb{B}^{n}\right)\right)>0$, contradicting $\operatorname{dim}_{H}\left(\Sigma_{2}\left(v, \mathbb{B}^{n}\right)\right)=0$ which follows from

$$
0 \leq \operatorname{dim}_{H}\left(\Sigma_{2}\left(u, \mathbb{B}^{n}\right)\right) \leq \operatorname{dim}_{H}\left(\Sigma_{2}(u, \Omega)\right)=0 .
$$

Therefore, $\Sigma_{2}(u, \Omega)$ consists of at most isolated points.

The part about the minimizing harmonic maps into a compact Riemannian manifold with metric $g$ is an immediate consequence of the above argument in that

$$
r \mapsto r^{2} \int_{B_{r}\left(x_{0}\right) \Subset \Omega}|\nabla u|_{g}^{2}
$$

is increasing; see e.g. [24, 19].

Remark 3.8. In his survey paper [24], Simon used the traditional "blowup" method to show that if $u$ is a minimizing harmonic map from $\Omega$ (which is allowed to be unbounded) into $\mathbb{S}^{2}$ then the singular set of $u$ (possibly being a global singular set) has its Hausdorff dimension at most $n-3$. Especially, this singular set is countably $(n-3)$-rectifiable. But note that rectifiability doesn't include sets of Hausdorff measure zero, so one could in Simon's situation have sets of fractional dimension, which by Theorem 2.2 one can not have.

3.6. Proof of Theorem 2.3. The proof uses Simon's characterization of the so-called tangent maps associated with each point $y \in \operatorname{sing}\left(u, \mathbb{B}^{n}\right)$; see also [24].

Each tangent map can be found by passing to the limit in the energy norm (for some possible subsequence) of $\rho \rightarrow 0$ in $u(y+\rho z) \rightarrow \phi_{y}(z)$. And then, it turns out, $\phi_{y}: \mathbb{R}^{n} \rightarrow \mathbb{S}^{m-1}$. Also, upon rotating $\phi_{y}$ one can write, as a limit,

$$
\phi_{0}\left(\frac{\hat{x}}{|\hat{x}|}\right), \hat{x} \in \mathbb{R}^{d} \text {, for some } d \geq 3 \text {. }
$$

Here $x=(\hat{x}, \bar{x}), \bar{x} \in \mathbb{R}^{n-d} \cdot \phi_{0}(\hat{\xi})$ is smooth on $|\hat{\xi}| \leq 1$. Upon considering

$$
I_{\rho}(z, y)=\int|z-x|^{1-n}|D(u(y+\rho x))| d x,
$$


we find two ways to evaluate the limit of $I_{\rho}(z, y)$ as $\rho \rightarrow 0$ : on the one hand,

$$
\begin{aligned}
\lim _{\rho \rightarrow 0} I_{\rho}(z, y) & =\lim _{\rho \rightarrow 0} \int|z-x|^{1-n} \rho|(D u)(y+\rho x)| d x \\
& =\lim _{\rho \rightarrow 0} \int|\rho z-w+y|^{1-n}|(D u)(w)| d w=I_{1}(|D u|)(y)
\end{aligned}
$$

on the other hand,

$$
\begin{aligned}
\lim _{\rho \rightarrow 0} I_{\rho}(z, y) & =\iint_{|\hat{x}| \leq 1}\left(|\hat{z}-\hat{x}|^{2}+|\bar{z}-\bar{x}|^{2}\right)^{(1-n) / 2}\left|D \phi_{0}\left(\frac{\hat{x}}{|\hat{x}|}\right)\right| d \hat{x} d \bar{x} \\
& =c \int_{|\hat{x}| \leq 1}|\hat{z}-\hat{x}|^{1-d}\left|\left(D \phi_{0}\right)\left(\frac{\hat{x}}{|\hat{x}|}\right)\right||\hat{x}|^{-1} d \hat{x}
\end{aligned}
$$

with $c$ being a constant. Thus, $I_{1}(|D u|)(y)$ diverges at $\hat{z}=0$ which corresponds to $y \in \operatorname{sing}\left(u, \mathbb{B}^{n}\right)$. We can clearly repeat this for any $y \in \operatorname{sing}\left(u, \mathbb{B}^{n}\right)$. Thus (2.4) holds because $I_{1}(|D u|)$ is smooth otherwise, due to the known smoothness of $|D u|$ off the $\operatorname{sing}\left(u, \mathbb{B}^{n}\right)$.

Remark 3.9. In accordance with [13, page 105] and [19, Corollary 2.2.8] one has that if $u$ is a $W^{1,2}$-minimizing harmonic map from $\mathbb{B}^{n}$ into $\mathbb{S}^{m-1}$ then

$$
\mathrm{S}_{2}\left(u, \mathbb{B}^{n}\right)=\operatorname{sing}\left(u, \mathbb{B}^{n}\right)=\left\{x_{0} \in \mathbb{B}^{n}: \lim _{r \rightarrow 0} r^{2} f_{B_{r}\left(x_{0}\right) \in \mathbb{B}^{n}}|\nabla u|^{2}>0\right\} .
$$

On the other hand, for $n \geq 4$ let $u(x, y)=x /|x|: \mathbb{R}^{3} \times \mathbb{R}^{n-3} \mapsto \mathbb{S}^{2}$. According to [19. Page 16], this is a minimizing harmonic map. It is not difficult to see that as a global singular set,

$$
\operatorname{sing}\left(u, \mathbb{R}^{n}\right)=\{0\} \times \mathbb{R}^{n-3}=\mathrm{S}_{2}\left(u, \mathbb{R}^{n}\right) \text { where } I_{1}(|\nabla u|)(x, y)=\infty,
$$

and $\operatorname{sodim}\left(\operatorname{sing}\left(u, \mathbb{R}^{n}\right)\right)=n-3$. It is worth noticing that one cannot get a local singular set of the type $\{0\} \times B_{R}(0)$ for some ball $B_{R}(0)$ in $\mathbb{R}^{n-3}$ by cutting off $u(x, y)$ and then passing to a limit. In fact, consider

$$
u_{j}(x, y)=\left(\frac{x}{|x|}\right) \phi_{j}(y) \quad \forall(x, y) \in \mathbb{R}^{3} \times \mathbb{R}^{n-3} \quad \& \quad j=1,2,3, \ldots,
$$

where

$$
\phi_{j}(y)=\phi_{j}(|y|)=\left\{\begin{array}{lll}
1 & \text { for } & |y| \leq 1-\frac{1}{j} \\
\text { linear } & \text { for } & 1-\frac{1}{j} \leq|y| \leq 1 \\
0 & \text { for } & |y| \geq 1 .
\end{array}\right.
$$

Of course, this function $\phi_{j}$ is only Lipchitz, but can be made better if needed - the conclusion is the same. Since $\left|\nabla \phi_{j}\right| \approx j$, one concludes

$$
\int_{x \in \mathbb{B}^{3}} \int_{y \in \mathbb{B}^{n-3}}\left|\nabla u_{j}(x, y)\right|^{2} \approx \int_{y \in \mathbb{B}^{n-3}} \int_{x \in \mathbb{B}^{3}}\left(|x|^{-2}+\left|\nabla \phi_{j}(y)\right|^{2}\right) \rightarrow \infty \text { as } j \rightarrow \infty .
$$


Note that $\lim _{j \rightarrow \infty} u_{j}(x, y)$ ought to be $u(x, y)$ (which is a minimizing harmonic map) on $\mathbb{B}^{3} \times \mathbb{B}^{n-3}$, the approximation of the constant function is no good in $W^{1,2}$, and $u_{j}$ is not a minimizing harmonic map. So, it is impossible to "bite off" a piece of a global singular set and to get a local singular set.

\section{REFERENCES}

[1] D. R. Adams, A note on Choquet integral with respect to Hausdorff capacity, in "Function Spaces and Applications," Lund 1986. Lecture Notes in Math. 1302, Springer-Verlag, 1988, pp. 115-124.

[2] D. R. Adams, A sharp inequality of J. Moser for higher order derivatives, Ann. Math. 128(1988)385-398.

[3] D. R. Adams and L. I. Hedberg, Function Spaces and Potential Theory. SpringerVerlag, Berlin Heidelberg, 1996.

[4] D. R. Adams and J. Xiao, Nonlinear analysis on Morrey spaces and their capacities, Indiana Univ. Math. J. 53(2004)1629-1663.

[5] D. R. Adams and J. Xiao, Morrey potentials and harmonic maps, Comm. Math. Phys. 308 (2011)439-456.

[6] D. R. Adams and J. Xiao, Morrey spaces in harmonic analysis, Ark. Mat. 50(2012)201-230.

[7] D. R. Adams and J. Xiao, Regularity of Morrey commutators, Trans. Amer. Math. Soc. 364(2012)4801-4818.

[8] A. Bensoussan and J. Frehse, Regularity results for nonlinear elliptic systems and applications, volume 151 of Applied Mathematical Sciences. Springer-Verlag, Berlin, 2002.

[9] F. Chiarenza and M. Frasca, Morrey spaces and Hardy-Littlewood maximal function, Rend. Mat. Appl. (7), 7(3-4)(1988)(1987)273-279.

[10] E. De Giorgi, Un esempio di estremali discontinue per un problema variazionale di tipo ellittico, Boll. UMI 4(1968)135-137.

[11] A. Friedman, Partial differential equations, Holt, Rinehart and Winston, Inc., New York, 1969.

[12] F. W. Gehring, The $L^{p}$-integrability of the partial derivatives of a quasiconformal mapping, Acta Math. 130(1973)265-277.

[13] M. Giaquinta, Multiple Integrals in the Calculus of Variations and Nonlinear Elliptic Systems, Ann. Math. Studies 105, Princeton University Press, Princeton, N.J., 1983.

[14] M. Giaquinta and E. Giusti, On the regularity of the minima of variational integrals, Acta Math. 148(1982)31-46.

[15] M. Giaquinta and E. Giusti, The singular set of the minima of certain quadratic functionals, Annali della Scuola Normale Superiore di Pisa 11:1(1984)45-55.

[16] E. Giusti and M. Miranda, Sulla regolarità delle soluzioni deboli di una classe di sistemi ellittici quasilineari, Arch. Rat. Mech. Anal. 31(1968)173-184.

[17] R. Hardt and F. Lin, The singular set of an energy minimizing map from $B^{4}$ to $S^{2}$, Manuscripta Math. 69(1990)275-289.

[18] A. Koshelev, Regularity problem for quasilinear elliptic and parabolic systems, Lecture Notes in Mathematics, 1614. Springer-Verlag, Berlin, 1995. xxii+255 pp.

[19] F. Lin and C. Wang. The analysis of harmonic maps and their heat flows. World Scientific Publishing Co. Pte. Ltd., Hackensack, NJ, 2008. 
[20] N. G. Meyers, An $L^{p}$ estimate for the gradient of solutions of second order elliptic divergence equations, Ann. Scuola Norm. Sup. Pisa (3) 17(1963)189-206.

[21] N. G. Meyers and A. Elcrat, Some results on regularity for solutions of non-linear elliptic systems and quasi-regular functions, Duke Math. J. 42(1975)121-136.

[22] C. B. Morrey, On the solutions of quasi-linear elliptic partial differential equations, Trans. Amer. Math. Soc. 43(1938)126-166.

[23] R. Schoen and S. T. Yau, Conformally flat manifolds, Kleinian groups and scalar curvature, Invent. Math. 92(1988)47-71.

[24] L. Simon, Singularities of geometric variational problems, In Nonlinear partial differential equations in differential geometry (Park City, UT, 1992), R. Hardt and M. Wolf ed., vol. 2 of IAS/Park City Math. Series, 185-223. Amer. Math. Soc., Providence, RI, 1996.

[25] C. T. Zorko, Morrey spaces, Proc. Amer. Math. Soc. 98(1986)586-592.

Department of Mathematics, University of Kentucky, Lexington, KY 40506-0027

E-mail address: dave@ms.uky.edu

Department of Mathematics and Statistics, Memorial University of Newfoundland, St. John's, NL A1C 5S7, CANAdA

E-mail address: jxiao@mun.ca 